\title{
Editorial. Altermondialisme(s) oublié(s)
}

Editorial. Forgotten Alterglobalization(s)

\section{Gülçin Erdi Lelandais}

\section{(2) OpenEdition}

\section{Journals}

\section{Édition électronique}

URL : http://journals.openedition.org/conflits/12213

DOI : 10.4000/conflits. 12213

ISSN : $1777-5345$

Éditeur :

CCLS - Centre d'études sur les conflits lilberté et sécurité, L'Harmattan

\section{Édition imprimée}

Date de publication : 20 juillet 2008

Pagination : 7-11

ISBN : 978-2-296-05909-2

ISSN : 1157-996X

Référence électronique

Gülçin Erdi Lelandais, " Editorial. Altermondialisme(s) oublié(s) », Cultures \& Conflits [En ligne], 70 | été 2008, mis en ligne le 10 septembre 2008, consulté le 30 mars 2021. URL : http://

journals.openedition.org/conflits/12213; DOI : https://doi.org/10.4000/conflits.12213 


\title{
Editorial. Altermondialisme(s) oublié(s)
}

\author{
Gülçin ERDI LELANDAIS
}

Gülçin Erdi Lelandais est docteure en sociologie de l'EHESS, chercheuse associée an Centre d'études sur les conflits et an Laboratoire de sciences sociales du politique (LaSSP). Ses travaux portent sur l'altermondialisme, la transnationalisation des monvements sociaux, la sociologie du risque et les mouvements sociaux et politiques en Turquie.

T a littérature sur la question de l'internationalisation des conflits mais aussi Lsur la transnationalisation des mouvements sociaux s'est considérablement développée depuis les années $1990{ }^{1}$. Toutefois, la plupart des recherches effectuées en la matière sont centrées sur l'altermondialisme dans des pays développés - notamment les Etats-Unis, les pays d'Europe du Nord, et en partie ceux d'Amérique latine - et néglige l'émergence et le développement de ce phénomène dans d'autres régions du monde, le Sud de la Méditerranée entre autres. Nous constatons également que les études sur l'altermondialisme ne se sont pas vraiment penchées sur les significations de ce phénomène dans cette aire géographique. Nous disposons de peu d'éléments sur l'implication des pays de cette région dans l'altermondialisme, ou sur son intensité et son apport ${ }^{2}$. De nombreux ouvrages publiés en France tentent certaines généralisations sur ces mouvements à partir du seul exemple des altermondialismes en Europe. Ce type d'approches entraîne le risque d'amener trop rapidement à des conclusions non nécessairement vérifiées par des analyses de l'autre côté de la Méditerranée. Par ailleurs, ce problème peut être accentué par l'absence de visibilité des organisations sur les zones géographiques précédemment citées. L'intérêt médiatique,

1. Fillieule O., Bennani-Chraïbi M., Résistances et protestations dans les sociétés musulmanes, Paris, Presses de Sciences-Po, 2003.

2 . Notre modeste contribution à cette littérature sur l'altermondialisme en Turquie constitue une des exceptions parmi d'autres recherches : Erdi Lelandais G., "Cosmopolites enracinés ». La transnationalité et la construction identitaire du mouvement altermondialiste. L'exemple de la Turquie, thèse de doctorat, EHESS, 2006, non publiée ; Cheynis E., «L'altermondialisme au prisme marocain », Critique internationale, 2005, n²7, pp. 177-191; Baykan B., Erdi Lelandais G., "Cross readings of the anti-globalisation movement in Turkey and beyond. Political culture in the making”, International Social Science Journal, n¹82, décembre 2004. 
mais aussi universitaire, est ainsi souvent dirigé vers les mouvements occidentaux, et lorsqu'une activité contestataire relativement importante apparaît dans un pays extra-européen de la Méditerranée, elle ne paraît pas susciter le même degré d'intérêt que ses homologues étrangers ${ }^{3}$.

Or, l'altermondialisme ne se développe pas qu'en Europe et ne possède pas toujours les caractéristiques présentées dans de nombreux ouvrages sur ce mouvement. Parmi la multitude de recherches collectives, ouvrages et colloques existants, nous trouvons encore peu d'études empiriques sur l'altermondialisme en Afrique et au Moyen-Orient par exemple. Tout semble ainsi porter à penser que ces zones géographiques sont exclues des préoccupations de la recherche en France. Lors de la préparation de ce numéro, nous avions le projet de réunir des chercheurs travaillant sur les pays extra-européens du bassin méditerranéen sur le sujet. Nous nous sommes aperçus que les mouvements sociaux dans ces pays ne sont quasiment pas étudiés, hormis les mouvements islamiques. On sait peu de choses, par exemple, sur des mouvements écologiques ou environnementaux. Nous pensons que ce vaste terrain est encore propice à de nombreuses explorations de la part des chercheurs en France.

Ainsi, au moment où les débats sur l'altermondialisme mais aussi sur les activités altermondialistes à travers le monde s'estompent, ou du moins semblent mis de côté, l'objectif de ce numéro est de relancer la discussion et de s'interroger sur les raisons de cet oubli tant médiatique qu'intellectuel, tout en nous intéressant à des mouvements altermondialistes qui semblent «oubliés »dans le champ d'étude de ce phénomène.

Le numéro que nous proposons a pour ambition d'offrir, à partir de l'autre côté de la Méditerranée, un panorama des mouvements sociaux. Ce panorama se veut représentatif, bien que non exhaustif, de la diversité de ces résistances, de leurs formes, de leur ampleur et de leurs effets.

Le sens et la motivation de l'altermondialisme diffèrent d'un pays à l'autre. Alors que l'altermondialisme en Europe est davantage lié à la lutte contre le néolibéralisme, il revêt notamment, dans certains pays du Sud de la Méditerranée, une forme de contestation vis-à-vis des fondements du champ politique, où l'on observe souvent des régimes politiques autoritaires et sécuritaires. Malgré cette différence sur le fondement des contestations, nous constatons dans toutes les formes de l'action collective liée à l'altermondialisme une prétention à l'universalité, dans la mesure où ses protagonistes se proclament défenseurs des droits humains, de la paix dans le monde et de la justice globale. Les articles du numéro nous montrent la présence et l'impor-

3. Voir Scholte J.A. (dir.), Démocratiser la mondialisation économique. Le rôle de la société civile, 2003, Rapport du CSGR, université de Warwick, consultable sur le site :

http://www.ifri.org/files/Economie/RapportScholte.pdf 
tance du niveau transnational dans quasiment toutes les organisations et revendications, quel que soit le pays dont celles-ci émanent.

On trouve également certains sujets de divergences communs à tous les pays au sein du mouvement altermondialiste. Le rapport entre le local et le global, l'appropriation de termes comme «impérialisme », le rôle de la région, l'identité collective transnationale, les relations dichotomiques entre les militants du «Nord » et ceux du «Sud », la crise de la gauche et la lutte pour la légitimité et la question leadership interne constituent presque partout des sujets de controverses internes au sein du mouvement.

Les contributions de ce numéro ont été choisies dans le souci d'apporter une pierre à la construction de ce double débat sur l'altermondialisme. D'un côté, nous avons souhaité présenter des mouvements et des causes « oubliés » tant par le mouvement altermondialiste lui-même que par la recherche le concernant. Ce faisant, nous avons également voulu refléter le point de vue des militants de ces mouvements à l'égard de l'altermondialisme européen. Lorsqu'on est un militant altermondialiste turc ou marocain, comment perçoit-on ce mouvement ? Que représente-il ? Que signifient le Forum social mondial ou le Forum social européen pour ce mouvement ? Chaque article aborde une ou plusieurs de ces interrogations, en offrant également un autre point de vue sur l'altermondialisme et ses représentations. Nous avons choisi de faire débuter le numéro par un article dressant un état des lieux général du mouvement altermondialisme (voir la contribution de Donatella della Porta dans ce volume). L'auteur y décrit avec précision l'histoire et l'état actuel du mouvement ainsi que ses contradictions intrinsèques, en mettant l'accent sur les oublis de la recherche sociologique sur ce mouvement. Malgré l'absence de recherches les concernant et la très faible visibilité des mouvements dans les pays du Sud, Della Porta souligne les constantes - souvent inattendues - de tous les mouvements altermondialistes. Le caractère transnational de ces luttes, comme nous avons souligné plus haut, au Nord comme au Sud, est plus que jamais d'actualité. En outre, malgré l'immaturité présupposée des luttes sociales au Sud, l'auteur nous montre que celles-ci, bien que très peu visibles, peuvent parfois avoir des effets plus conséquents en termes de changements sociaux et politiques. Le Mouvement pour une justice globale et contre la guerre en Irak en Turquie, qui a fortement contribué à empêcher l'envoi de troupes turques dans ce pays, ou encore l'arrivée au pouvoir d'Evo Morales, issu du mouvement indien en Bolivie, en sont des exemples significatifs.

Le mouvement altermondialiste mène également ces débats en son propre sein et s'interroge sur les possibilités de diversifier les représentations. Ainsi, Raphaël Canet nous livre une comparaison, quasiment inédite, entre différents forums sociaux, du niveau local au niveau mondial. Il nous montre les efforts faits au sein du mouvement altermondialiste pour inclure le plus de mouvements possibles en son sein. Ce faisant, il renverse le processus d'étude du Forum social mondial (FSM) : au lieu de focaliser l'analyse sur les processus 
d'élaboration des forums, il s'intéresse à la construction des initiatives locales et régionales à partir de l'expérience du FSM. Cette analyse comparative nous montre l'importance des initiatives régionales, de la présence d'une marge démocratique pour l'expression sociale et politique et d'une société civile établie afin de pouvoir s'organiser.

Dans ce cadre, les articles suivants fournissent chacun une étude de cas précis confirmant les constats que nous venons de faire. Deux articles, celui d'Eric Cheynis et celui de Gülçin Erdi Lelandais, présentent les mouvements altermondialistes dans deux pays musulmans: en Turquie et au Maroc. Timothy Peace décrit la participation des organisations musulmanes issues de deux pays de l'Union européenne, à savoir la France et l'Angleterre. Ces trois articles nous fournissent un aperçu relativement complet de la participation musulmane, sujet à controverse, dans l'altermondialisme européen, en articulant l'extérieur et l'intérieur.

Si l'altermondialisme présente des caractéristiques différentes selon les pays, comme le reflètent les articles ici rassemblés, certaines grandes lignes communes se dégagent néanmoins autour de la manière de le concevoir. Ainsi, l'altermondialisme en Turquie et au Maroc présente parfois des particularités similaires concernant les profils et les reconversions militantes. Gülçin Erdi Lelandais analyse en quoi l'altermondialisme en Turquie est le fruit de l'investissement de militants intégrés dans des réseaux transnationaux de militantisme, que ce soit dans le domaine politique avec le groupe Antikapitalist ou syndical avec le groupe de travail contre l'AMI, Anti-MAI. Elle décrit l'altermondialisme comme une nouvelle cause dans le champ militant, due aux transformations observées au cours des vingt dernières années dans le champ politique turc. D'après l'auteur, l'altermondialisme en Turquie présente des traits d'un mouvement anti-système et s'oppose, dans ce sens, aux positionnements réformistes de certaines organisations comme ATTAC en Europe. Il contribue également à l'apparition d'un nouveau type de militants refusant toute forme d'obéissance à une organisation politique, pourtant caractéristique majeure des organisations de gauche en Turquie.

Eric Cheynis nous livre le même constat, cette fois concernant le positionnement des militants dans l'altermondialisme au Maroc. Il souligne la dimension transnationale fortement influente dans les trajectoires des militants altermondialistes et montre le rôle décisif de la socialisation internationale, comme dans le cas de la Turquie, pour l'implication dans l'altermondialisme. Cheynis décrit les différentes motivations au sein de l'altermondialisme au Maroc, composé en deux pôles opposés. D'un côté, le secteur associatif avec des moyens financiers non négligeables, incarné par l'AMSED ; de l'autre le pôle politique représenté par ATTAC Maroc, avec des moyens limités. En partant de l'analyse des militants de ces deux secteurs, l'auteur montre que la cause altermondialiste, représentant une alternative politique démocratique pour certains militants 
déçus par les organisations issues de l'extrême gauche, peut constituer pour d'autres une opportunité de carrière professionnelle dans le secteur associatif.

Mais l'altermondialisme, au-delà d'une opportunité de reconversion de la gauche radicale, représente également un espace pour différentes luttes identitaires, en Turquie et au Maroc. L'exemple le plus flagrant est la présence d'organisations musulmanes œuvrant pour la possibilité de porter le voile islamique dans l'enseignement supérieur en Turquie. Ce sujet constitue justement un point de discorde à l'intérieur de la mouvance altermondialiste, source de bipolarisation. Alors que la présence d'associations musulmanes est considérée comme normale en Turquie, car, comme l'extrême gauche, l'islamisme en tant que courant politique a toujours été réprimé par les kémalistes dans ce pays, les altermondialistes français y sont plus que réticents, au nom du principe de läcité. Timothy Peace met au jour ce clivage au sein de l'altermondialisme en comparant la France et la Grande-Bretagne. Ce type d'étude comparative n'avait pas encore été réalisé et montre comment la définition d'un autre monde, les moyens d'y accéder et le choix des « compagnons de route » pour y parvenir ne sont pas du tout perçus de la même manière, même dans les mouvements altermondialistes du Nord de l'Europe.

Enfin, Marie-Emmanuelle Pommerolle et Johanna Siméant complètent ces études de cas par l'analyse de la participation africaine, si fragile et oubliée, dans les forums sociaux. Les auteurs évoquent dans leur article deux phénomènes essentiels : d'une part le transnationalisme des réseaux altermondialistes africains et d'autre part, la relation complexe que ceux-ci entretiennent avec les militants des pays du Nord. Dans ce sens, les auteurs mettent l'accent sur un point fondamental et peu analysé dans les études sur l'altermondialisme, la division du travail au sein du militantisme transnational, accompagnant leur réflexion d'une analyse sur la capacité et la légitimité à parler «au nom de l'Afrique ». Comme dans le cas du Maroc, l'altermondialisme semblerait en Afrique, d'après les auteurs, se regrouper en deux pôles : les ONG dirigées par «des élites intellectuelles réformistes » et un pôle social visant davantage une transformation sociale et politique.

Ces similitudes inattendues entre les différents mouvements altermondialistes, que nous trouvons d'ailleurs également dans la plupart des mouvements en Europe et en Amérique latine (voir l'article d'Odaci Luiz Coradini dans ce numéro), soulignent une fois encore le débat prépondérant au sein de cette mouvance : si le caractère réformiste du mouvement domine, la réémergence d'une conscience de classe autour de luttes politiques radicales trouvant ses référents dans différentes formes du marxisme est également aujourd'hui une réalité pour la cause altermondialiste. 